\title{
Full and Reduced Order Extended Kalman Filter for Speed Estimation in Induction Motor Drives: A Comparative Study
}

\author{
Américo Vicente Leite \\ Instituto Politécnico de Bragança \\ Escola Superior de Tecnologia e de Gestão \\ Bragança, Portugal \\ Email: avtl@ipb.pt
}

\author{
Rui Esteves Araújo \\ Universidade do Porto \\ Faculdade de Engenharia \\ Porto, Portugal \\ Email: raraujo@fe.up.pt
}

\author{
Diamantino Freitas \\ Universidade do Porto \\ Faculdade de Engenharia \\ Porto, Portugal \\ Email: dfreitas@fe.up.pt
}

\begin{abstract}
This paper presents a comparative study between a new approach for robust speed estimation in induction motor sensorless control, using a reduced order Extended Kalman Filter (EKF), and the one performed by the full order EKF. The new EKF algorithm uses a reduced order state-space model that is discretized in a particular and innovative way. In this case only the rotor flux components are estimated, besides the rotor speed, while the full order EKF also estimates stator current components. This new approach strongly reduces the execution time and simplifies the tuning of covariance matrices. The performance of speed estimation using both EKF techniques is compared with respect to computation effort, tuning of the algorithms, speed range including low speeds, load torque conditions and robustness relatively to motor parameter sensitivity.
\end{abstract}

\section{INTRODUCTION}

Due to lower cost and greater reliability without mounting problems, sensorless control methods have been making remarkable developments in the most recent years [1], [2]. Speed estimation methods are being used that avoid the speed measurement set-up and commercial sensorless vector-controlled drives are already available.

There are several speed estimation techniques in the literature which are generally classified in [3] as follows: slip calculation; direct synthesis from state equations; model reference adaptive system (MRAS) [4]; speed adaptive flux observer; slot harmonics; injection of auxiliary signal on salient rotor to improve the estimation at low speed [5] and extended Kalman filter (EKF) based methods, [6], [7].

A recent effort in research on sensorless drive control of standard induction motors has been the estimation of rotor speed from the measurement of stator voltages and currents using either the MRAS [4], [8] or the EKF [6], [7] and [9].

This work presents a comparative study between full and reduced order EKF speed estimation techniques for induction motor sensorless control, relatively to computation effort, tuning of the algorithms, speed range including low speeds, load torque conditions and robustness with respect to parameter variation. Firstly, a new approach for real-time estimation of mechanical speed and rotor flux components using a reduced order EKF is presented and secondly, this approach is compared with the full order EKF that also estimates stator current components, besides the estimation of the speed itself and rotor flux components like in [6] and [7]. Instead of these flux components, the rotor current ones are estimated in [9] and the magnetizing current is estimated in [10].

In the new approach only the rotor flux $d q$ components are estimated, besides the rotor speed itself. Consequently, practical and important improvements are achieved with respect to the well known drawbacks associated to the EKF, like the computational effort for real-time applications, the complexity and the hard tuning of the covariance matrices. In fact, with the $3^{\text {rd }}$ order EKF that is obtained, the dimension of all matrices of the algorithm becomes small enough from a practical point of view. On the one hand the computational effort is smaller and, on the other hand, the complexity is reduced since the tuning of the algorithm becomes simpler, mainly due to the lower dimension of the state vector. Thus, the genetic algorithm proposed in [6] is not needed any more. Furthermore, if needed, a $2^{\text {nd }}$ order approximation becomes practicable and not heavy with the special discretization process proposed by the authors in this work.

New improvements as well as important and practical aspects are described in this paper. They contribute strongly to reduce the execution time of the new algorithm without difficulties related to the tuning of covariance matrices.

The reduced order EKF algorithm has been implemented and validated in real-time operation and the performance of speed estimation using both EKF techniques are compared with respect to computation effort, tuning of the algorithms, parameter variation and other important aspects.

\section{FULL AND REDUCED ORDER MODEL STRUCTURES FOR THE EXTENDED KALMAN FILTER}

The well-known and established $d q$ dynamic model of a squirrel-cage induction motor is represented by its stator and rotor space phasors voltage equations and stator and rotor flux expressed in terms of stator and rotor currents space phasors [11].

Considering the induction motor equations in the stator reference frame, and eliminating stator flux and rotor currents space phasors followed by some algebraic manipulations, the following state-space model structure can be obtained, where $\dot{x}$ means $d x / d t$ :

$\left[\begin{array}{c}\dot{i}_{s d}^{s} \\ \dot{i}_{s q}^{s} \\ \dot{\psi}_{r d}^{s} \\ \dot{\psi}_{r q}^{s}\end{array}\right]=\left[\begin{array}{cccc}a & 0 & b & c \\ 0 & a & -c & b \\ d & 0 & -\tau_{r}^{-1} & -\omega \\ 0 & d & \omega & -\tau_{r}^{-1}\end{array}\right]\left[\begin{array}{c}i_{s d}^{s} \\ i_{s q}^{s} \\ \psi_{r d}^{s} \\ \psi_{r q}^{s}\end{array}\right]+\left[\begin{array}{cc}L_{s}^{-1} & 0 \\ 0 & L_{s}^{\prime-1} \\ 0 & 0 \\ 0 & 0\end{array}\right]\left[\begin{array}{c}u_{s d}^{s} \\ u_{s q}^{s}\end{array}\right],(1 \mathrm{a})$ 


$$
y=\left[\begin{array}{ll}
i_{s d}^{s} & i_{s q}^{s}
\end{array}\right]^{T}=\left[\begin{array}{llll}
1 & 0 & 0 & 0 \\
0 & 1 & 0 & 0
\end{array}\right]\left[\begin{array}{llll}
i_{s d}^{s} & i_{s q}^{s} & \psi_{r d}^{s} & \psi_{r q}^{s}
\end{array}\right]^{T},
$$

The electrical parameters $\tau_{r}, L_{s}^{\prime}, L_{M}$ and $R_{s}$ represent the rotor time constant, stator transient inductance, mutual inductance and stator resistance, respectively, and $\omega$ is the electrical rotor speed in $\mathrm{rad} / \mathrm{s}$. The variables $u_{s d, q}^{s}$ and $i_{s d, q}^{s}$ are the stator voltage and current $d q$ components, respectively, and $\psi_{r d, q}^{s}$ are the referred rotor flux ones. The other parameters of (1) are given by:

$a=-L_{s}^{\prime-1}\left(R_{s}+L_{M} \tau_{r}^{-1}\right), b=L_{s}^{\prime-1} \tau_{r}^{-1}, c=\omega L_{s}^{\prime-1}, d=L_{M} \tau_{r}^{-1}$

The state-space model (1) is the type of model structure used for speed estimation using full order EKF, like in [6] and [7]. The state vector of the state-space model (1),

$$
x(t)=\left[\begin{array}{llll}
i_{s d}^{s}(t) & i_{s q}^{s}(t) & \psi_{r d}^{s}(t) & \psi_{r q}^{s}(t)
\end{array}\right]^{T},
$$

also includes the measured stator current $d q$ components besides the rotor flux $d q$ components that must be jointly estimated with the rotor speed. The state-vector (2) is then extended to rotor speed that becomes a new state variable.

Instead of the resulting $5^{\text {th }}$ order model, a reduced order state-space model structure is now derived for speed estimation. It is obtained rewriting (1a), in a different way but keeping the general form of $\dot{x}(t)=A x(t)+B u(t)$, and $y(t)=C x(t)$, resulting, respectively, as follows:

$$
\begin{aligned}
& {\left[\begin{array}{c}
\dot{\psi}_{r d}^{s} \\
\dot{\psi}_{r q}^{s}
\end{array}\right]=\left[\begin{array}{cc}
-\tau_{r}^{-1} & -\omega \\
\omega & -\tau_{r}^{-1}
\end{array}\right]\left[\begin{array}{c}
\psi_{r d}^{s} \\
\psi_{r q}^{s}
\end{array}\right]+\left[\begin{array}{cc}
L_{M} \tau_{r}^{-1} & 0 \\
0 & L_{M} \tau_{r}^{-1}
\end{array}\right]\left[\begin{array}{c}
i_{s d}^{s} \\
i_{s q}^{s}
\end{array}\right],} \\
& {\left[\begin{array}{c}
u_{s d}^{s}-\left(R_{s}+L_{M} \tau_{r}^{-1}\right) i_{s d}^{s}-L_{s}^{\prime} i_{s d}^{s} \\
u_{s q}^{s}-\left(R_{s}+L_{M} \tau_{r}^{-1}\right) i_{s q}^{s}-L_{s}^{\prime} i_{s q}^{s}
\end{array}\right]=\left[\begin{array}{cc}
-\tau_{r}^{-1} & -\omega \\
\omega & -\tau_{r}^{-1}
\end{array}\right]\left[\begin{array}{c}
\psi_{r d}^{s} \\
\psi_{r q}^{s}
\end{array}\right] .}
\end{aligned}
$$

Equations (3a) and (3b) are the reduced state equation and the new output equation, respectively. Thus, the state vector and the input and output vectors become, respectively:

$$
\begin{gathered}
x(t)=\left[\begin{array}{ll}
\psi_{r d}^{s}(t) & \psi_{r q}^{s}(t)
\end{array}\right]^{T}=\left[\begin{array}{ll}
x_{1}(t) & x_{2}(t)
\end{array}\right]^{T}, \\
u(t)=\left[\begin{array}{ll}
i_{s d}^{s}(t) & i_{s q}^{s}(t)
\end{array}\right]^{T}=\left[\begin{array}{ll}
u_{1}(t) & u_{2}(t)
\end{array}\right]^{T}, \\
y(t)=\left[\begin{array}{ll}
y_{1}(t) & y_{2}(t)
\end{array}\right]^{T},
\end{gathered}
$$

with the outputs $y_{1}$ and $y_{2}$ given by:

$$
\begin{aligned}
& y_{1}=u_{s d}^{s}-\left(R_{s}+L_{M} \tau_{r}^{-1}\right) i_{s d}^{s}-L_{s}^{\prime} \dot{i}_{s d}^{s}, \\
& y_{2}=u_{s q}^{s}-\left(R_{s}+L_{M} \tau_{r}^{-1}\right) i_{s q}^{s}-L_{s}^{\prime} \dot{i}_{s q}^{s} .
\end{aligned}
$$

\section{A. Full order EKF}

For speed estimation using a full order EKF, the model structure given by (1a) and (1b) is discretized directly using the following and well known relationships [12]:

$A_{d}=I+A T_{s}+\frac{A^{2} T_{s}^{2}}{2 !}+\frac{A^{3} T_{s}^{3}}{3 !}+\cdots, B_{d}=B T_{s}+\frac{A B T_{s}^{2}}{2 !}+\frac{A^{2} B T_{s}^{3}}{3 !}+\cdots$

Furthermore, the state vector (2) is extended to rotor speed, as follows,

$$
x_{e}(k)=\left[\begin{array}{lllll}
i_{s d}^{s}(k) & i_{s q}^{s}(k) & \psi_{r d}^{s}(k) & \psi_{r q}^{s}(k) & \omega(k)
\end{array}\right]^{T}
$$

It is assumed that the real speed varies like a random walk, according to:

$$
\omega(k+1)=\omega(k)+r_{\omega}(k)
$$

with $r_{\omega}(k)$ white Gaussian noise. Considering the discrete output matrix $C_{d}=C$, and applying an approximation of (6) to (1a) a $5^{\text {th }}$ order nonlinear state-space model is obtained. Then the denominated full order EKF can be used. Normally the approximation to the linear terms of (6) is used, which is acceptable if the sampling time $T_{s}$ is small enough. This procedure is similar to compute the derivatives in (1a) by means of the well-known Euler's formula.

\section{B. Reduced order EKF}

For the reduced order model (3), proposed in this work, an innovative discretization process is applied. Although the state equation (3a) had been discretized using (6) the discrete output equation corresponding to (3b) was obtained directly with the first derivative of the stator current components computed not by means of Euler's approximation but using a filter of the type [13]:

$$
\left.\dot{i}_{s d, q}\right|_{t=t_{k}} \approx \frac{1}{T_{s}} \sum_{i=0}^{n-1} C_{i} i_{s d, q}\left(t_{k}-i T_{s}\right) .
$$

It is only required that the delay introduced by the filter be similar to the one introduced by the approximation used in (6). For a sampling frequency of $5 \mathrm{kHz}$ the approximations:

$$
A_{d} \approx I+A T_{s} \text { and } B_{d} \approx B T_{s},
$$

$\left.i_{s d, q}\right|_{t=t_{k}} \approx \frac{11 i_{s d, q}(k)-18 i_{s d, q}(k-1)+9 i_{s d, q}(k-2)-2 i_{s d, q}(k-3)}{6 T_{s}}$,

have been used successfully with very good results. The matrices $A_{d}$ and $B_{d}$ are the discrete forms of $A$ and $B$. The state vector (4a) is extended to the rotor speed as a new state variable to be jointly estimated with the rotor flux components and the extended state vector is given by:

$x_{e}(k)=\left[\begin{array}{lll}\psi_{r d}^{s}(k) & \psi_{r q}^{s}(k) & \omega(k)\end{array}\right]^{T}=\left[\begin{array}{lll}x_{e_{1}}(k) & x_{e_{1}}(k) & x_{e 3}(k)\end{array}\right]^{T}$,

assuming that the real speed varies according to (8). Applying (10a) and (10b) to (3a) and (3b), respectively, and adding the equation (8), the following stochastic nonlinear reduced order model is obtained:

$$
x_{e}(k+1)=f\left(x_{e}(k), u(k), r_{s}(k)\right)=
$$




$$
\begin{aligned}
& =\left\{\begin{array}{l}
\left(1-\tau_{r}^{-1} T_{s}\right) x_{e 1}(k)-x_{e 3}(k) x_{e 2}(k) T_{s}+L_{M} \tau_{r}^{-1} T_{s} u_{1}(k)+r_{s 1}(k) \\
x_{e 3}(k) x_{e 1}(k) T_{s}+\left(1-\tau_{r}^{-1} T_{s}\right) x_{e 2}(k)+L_{M} \tau_{r}^{-1} T_{s} u_{2}(k)+r_{s 2}(k) \\
x_{e 3}(k)+r_{\omega}(k)
\end{array}\right. \\
& \hat{y}=\left[\begin{array}{ll}
\hat{y}_{1}(k) & \hat{y}_{2}(k)
\end{array}\right]^{T}= \\
& =\left\{\begin{array}{l}
h_{1}\left(x_{e}(k), r_{m}(k)\right) \\
h_{2}\left(x_{e}(k), r_{m}(k)\right)
\end{array}=\left\{\begin{array}{l}
-\tau_{r}^{-1} x_{e 1}(t)-x_{e 3}(t) x_{e 2}(t)+r_{m 1}(k) \\
x_{e 3}(t) x_{e 1}(t)-\tau_{r}^{-1} x_{e 2}(t)+r_{m 2}(k)
\end{array}\right.\right. \\
& y=\left\{\begin{array}{l}
y_{1}(k) \\
y_{2}(k)
\end{array}=\left\{\begin{array}{l}
u_{s d}^{s}(k)-\left(R_{s}+L_{M} \tau_{r}^{-1}\right) i_{s d}^{s}(k)-\left.L_{s}^{\prime} \dot{i}_{s d}\right|_{t=t_{k}} \\
u_{s q}^{s}(k)-\left(R_{s}+L_{M} \tau_{r}^{-1}\right) i_{s q}^{s}(k)-\left.L_{s}^{\prime} \dot{i}_{s q}\right|_{t=t_{k}}
\end{array}\right.\right.
\end{aligned}
$$

The reduced order EKF can now be applied to the nonlinear model described by (12) to (14) and the state vector is estimated in order to minimize the prediction error, $\varepsilon(k)=y(k)-\hat{y}(k)$.

\section{Comparison relatively to the dimension of the matrices}

When the full order EKF based on (1) is used, the dimension of the state vector (7) is $5 \times 1$ and the system, distribution and output matrices, $\left(A_{d}, B_{d}\right.$ and $C_{d}$ ) have dimensions of $5 \times 5,5 \times 2$ and $2 \times 5$, respectively. Furthermore, the system noise covariance matrix $(Q)$ has dimension of $5 \times 5$, that is to say we have 5 diagonal elements to be tuned. On the other hand, the gradient matrices $F$ and $H$ of the EKF algorithm presented in the appendix section have dimensions of $5 \times 5$ and $2 \times 5$, respectively. When the reduced order EKF based on (3) is used, the dimension of the new state vector (11) is $3 \times 1$ and those matrices have the following dimensions: $3 \times 3,3 \times 2$, $2 \times 3,3 \times 3,3 \times 3$ and $2 \times 3$, respectively. Consequently, the global computational effort is reduced and we need to tune only 3 elements in the diagonal system noise covariance matrix, instead of 5. Actually, we have to tune just 2 elements since, among the 3 ones, 2 of them can be equal.

Another important advantage of the proposed reduced order model is that the matrices $A, B$ and $C$, do not depend neither on the stator parameters nor on the first derivative of the stator current components as can be seen in description (3). These parameters and derivatives only appear in the virtual outputs calculated according to (14). So, we can expect a good robustness with respect to these drawbacks since they can be taken into account by tuning conveniently the measurement noise covariance matrix. This means that the errors due to these factors are converted to a lack of confidence in the measures.

\section{EXPERIMENTAL RERSULTS}

Both EKF algorithms have been investigated with simulation tests by means of Simulink. The real-time validation and comparison have been performed using the dSPACE ACE kit based on the DS1 103 controller board. It was used a $3 \mathrm{~kW}, 400 \mathrm{~V}, 6.3 \mathrm{~A}, 50 \mathrm{~Hz}, 1430 \mathrm{rpm}$, squirrel-cage induction motor (ref. LSFMV100) with an incremental encoder and loaded by a programmable powder brake (ref. FP15/30) from Leroy Somer, to achieve the experimental results shown in this section. The focus of this work is the comparison of both EKF identification algorithms which were widely tested in real-time operation using the dSPACE kit. The induction motor has been controlled by an ABB frequency converter of the ACS600's family, with open loop speed control.

For both algorithms the speed estimation is based on the stator voltage and current $d q$ components. In the practical implementation, these signals, expressed in the stator reference frame, are filtered with analogue elliptic low-pass pre-filters of fifth order with a $500 \mathrm{~Hz}$ cutoff frequency. A sampling frequency of $5 \mathrm{kHz}$ was used, and the speed estimation is computed with the same cadency.

The electrical parameters of the induction motor were previously estimated by means of the identification techniques proposed by the same authors in the references [14] and [15]. The estimated values of the parameters are the following: $\tau_{r}=160 \mathrm{~ms}, L_{s}^{\prime}=10 \mathrm{mH}, L_{M}=200 \mathrm{mH}$ and $R_{s}=2.4 \Omega$.

\section{A. Some implementation details}

The state variables have very different magnitudes since the rotor flux has amplitude close to one and the nominal electrical speed is about $300 \mathrm{rad} / \mathrm{s}$. This happens in the extended sate vector (11) used by the reduced order EKF. In the implementation scheme, within a simulink block, the third state, corresponding to the rotor speed, was scaled as follows:

$$
x_{e 3}(k)=K_{\omega} \omega(k), \text { with } K_{\omega}=0.0032 .
$$

The problems related to these different sizes are twofold: firstly, numerical problems can arise and, secondly, the states with reduced size can be estimated with significant errors. On the other hand, the speed scaling according to (15) gives another advantage of practical interest. Although with different dynamics, the state variables have approximately the same magnitude and the tuning of the diagonal elements of the system covariance matrix become similar. Moreover, since the two components of the rotor flux have the same magnitude and dynamics, only two elements of the system covariance matrix $(Q)$ must be tuned:

$$
Q=\operatorname{diag}\left(\left[\begin{array}{lll}
Q_{11} & Q_{22} & Q_{33}
\end{array}\right], 0\right), \text { with } Q_{33} \approx Q_{22}=Q_{11} .
$$

In (16) diag defines a diagonal matrix, with $Q_{i j}=0$.

For the full order EKF, the extended state vector (7) also includes the stator current $d q$ components which should be equally scaled being an additional requirement. In this case the following scaling procedure was used:

$$
x_{e_{1}}(k)=K_{i} i_{s d}^{s}(k), x_{e_{2}}(k)=K_{i} i_{s q}^{s}(k), x_{e_{5}}(k)=K_{\omega} \omega(k) .
$$

In (17) the scaling factors $K_{i}$ and $K_{\omega}$ are equal to 0.2 and 0.0032 , respectively. Therefore, the tuning of the 
system covariance matrix becomes more difficult since it has now five elements:

$$
Q=\operatorname{diag}\left(\left[\begin{array}{lllll}
Q_{11} & Q_{22} & Q_{33} & Q_{44} & Q_{55}
\end{array}\right], 0\right),
$$

with $Q_{55} \approx Q_{22}=Q_{11} \approx Q_{44}=Q_{33}$.

Furthermore, in both algorithms, the measurement noise covariance matrix has been fixed as follows:

$$
R_{m}=\operatorname{diag}\left(\left[\begin{array}{ll}
R_{11} & R_{22}
\end{array}\right], 0\right) \text {, with } R_{22}=R_{11}=1 .
$$

If the measurement noise covariance matrix is normalized as in (19), the tuning of both EKF algorithms will be simplified since it will be achieved just by means of the system covariance matrices (16) and (18), respectively, for reduced and full EKF. The above considerations are very important because the EKF tuning is performed in an ad hoc way and becomes much simpler with the reduced order EKF than with the full order one.

\section{B. Initialization of the EKF algorithms}

The full order EKF was initialized as follows:

$$
\begin{gathered}
x_{e}(0)=\left[\begin{array}{lllll}
0.1 & 0.1 & 0 & 0 & 0
\end{array}\right]^{T}, \\
P(0)=\operatorname{diag}\left(\left[\begin{array}{lllll}
10^{-8} & 10^{-8} & 10^{-8} & 10^{-8} & 0
\end{array}\right], 0\right), \\
Q(0)=Q=\operatorname{diag}\left(\left[\begin{array}{lllll}
10^{-6} & 10^{-6} & 10^{-5} & 10^{-5} & 10^{-6}
\end{array}\right], 0\right),
\end{gathered}
$$

and the reduced order EKF initialization becomes simpler and was performed as follows:

$$
\begin{gathered}
x_{e}(0)=\left[\begin{array}{lll}
0 & 0 & 0
\end{array}\right]^{T}, \\
P(0)=\operatorname{diag}\left(\left[\begin{array}{lll}
10^{-8} & 10^{-8} & 0
\end{array}\right], 0\right), \\
Q(0)=Q=\operatorname{diag}\left(\left[\begin{array}{lll}
10^{-6} & 10^{-6} & 10^{-7}
\end{array}\right], 0\right) .
\end{gathered}
$$

The effect of the state covariance matrix $(P)$ of prediction error, given by (20b) and (21b) in case of full and reduced order EKF, respectively, is not important after the first instants. Moreover, its initialization is not critical in both EKF algorithms.

\section{Transient versus steady-state performance}

The system covariance matrices $Q$, given by (20c) and (21c), play an important role in the behavior of the algorithms mainly the elements $Q_{33}$ in (21c) and $Q_{55}$ in (20c), which control directly the gain of the EKF algorithms, relatively to the estimated speed. Fig. 1 and 2 show the effect in the estimated speed when tuning $Q_{55}$ and $Q_{33}$ which are used in full and reduced order EKF, respectively.

In fig. 1, the rotor speed was estimated using the reduced order EKF with:

$$
Q=\operatorname{diag}\left(\left[\begin{array}{lllll}
10^{-6} & 10^{-6} & 10^{-5} & 10^{-5} & 10^{-6}
\end{array}\right], 0\right)
$$

In fig. 2 the element $Q_{55}$ of (22) was changed from $10^{-6}$ to $10^{-5}$. With respect to the reduced order EKF, the rotor speed was estimated with:

$$
Q=\operatorname{diag}\left(\left[\begin{array}{lll}
10^{-6} & 10^{-6} & 10^{-7}
\end{array}\right], 0\right)
$$

In fig. 2 the element $Q_{33}$ of (23) was changed from $10^{-7}$ to $10^{-6}$. The main conclusions from the analysis of fig. 1 and 2 are the following: firstly, the performance of both EKF algorithms is very similar and, secondly, it is a compromise between transient and steady-state operation of the motor. This means that the error in the estimated speed can be very low in transient conditions being higher in steady-state operation and vise-versa.
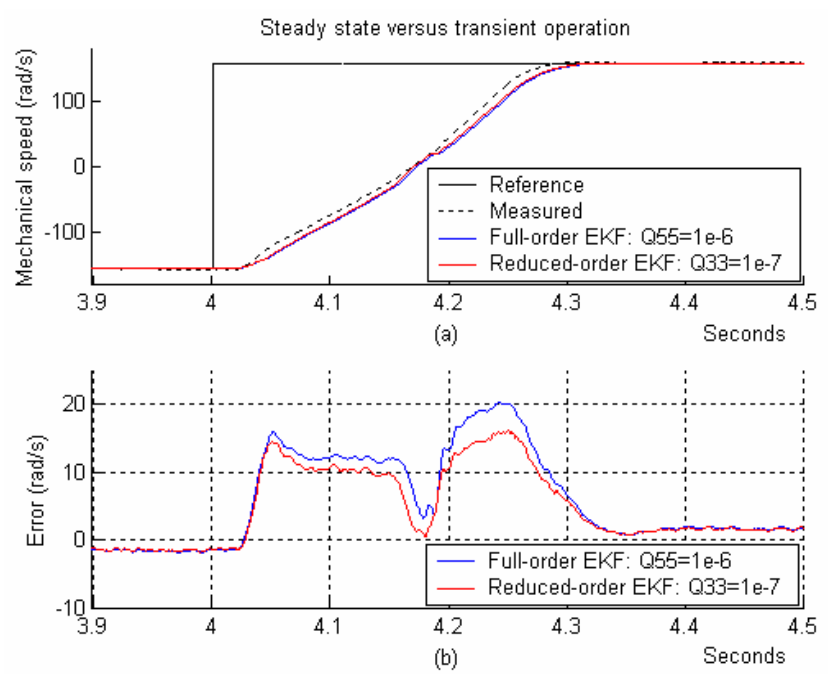

Fig. 1. Comparison of the transient versus steady-state performance of the full and reduced order EKF with $Q_{55}=10^{-6}$ and $Q_{33}=10^{-7}$, respectively. (a) reference, measured and estimated speeds in a speed reversal from -1500 to $+1500 \mathrm{rpm}$. (b) speed error in $\mathrm{rad} / \mathrm{s}$.
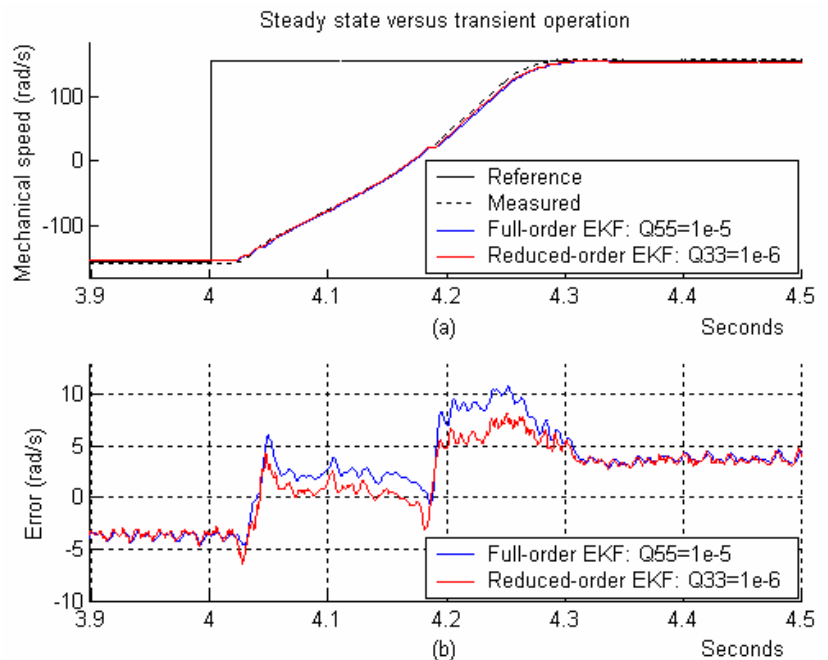

Fig. 2. The same of fig. 1 but with $Q_{55}=10^{-5}$ and $Q_{33}=10^{-6}$.

\section{Comparison of the sensitivity to parameter variation}

A series of tests were achieved in order to compare the behavior of the algorithms with respect to parameter variation. The performance depends on speed range and 
load torque but the main conclusion is that it is similar for both algorithms. At high speeds, above some hundreds of $\mathrm{rpm}$, the speed error is reduced even with full load as shown in fig. 3(a) to 3(d). The electrical parameters were varied in a range of $\pm 50 \%$ around their estimated values, at a constant speed of $1500 \mathrm{rpm}$, with nominal torque.

The higher errors happen under full load at low speeds as shown in fig. (4). Once again, the electrical parameters were varied in a range of $\pm 50 \%$ but at a constant speed of 100 $\mathrm{rpm}$. The magnitude of the errors in the estimated speed is similar for both algorithms. In both fig. 3 and 4, the errors were filtered with a non-causal filter in order to guarantee the legibility of the comparison since the errors are very noisy, and to avoid the delay in the filtered errors.

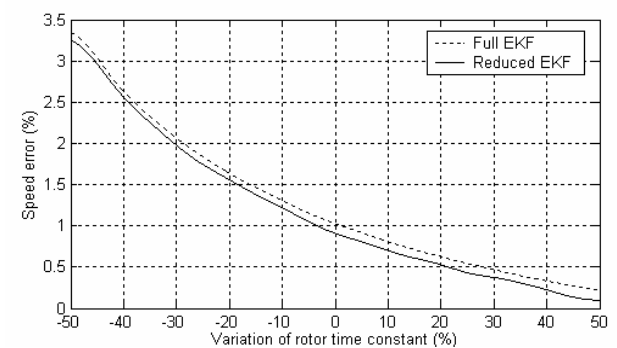

(a)

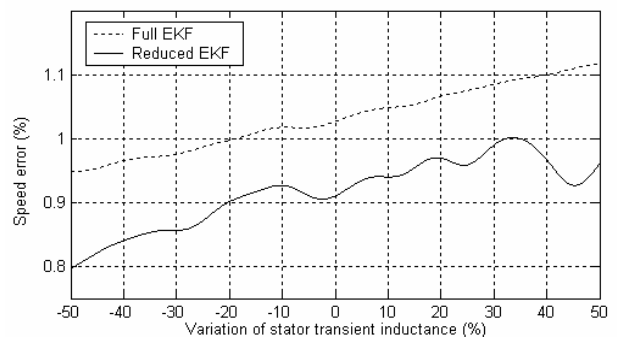

(b)

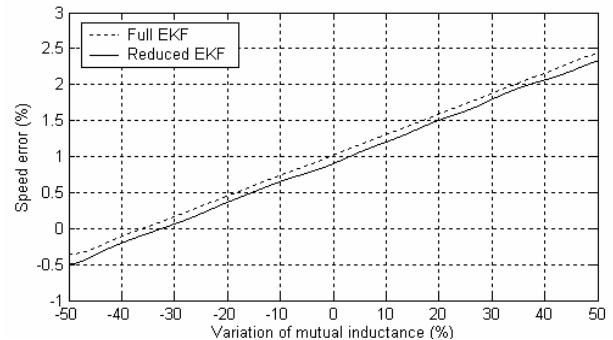

(c)

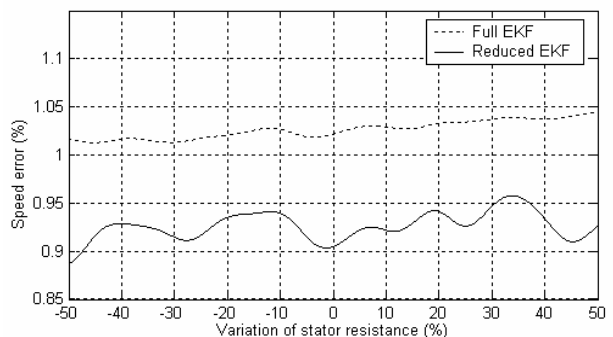

(d)

Fig. 3. Comparison of full and reduced order EKF at $1500 \mathrm{rpm}$, under full load, when the electrical parameters are varied from $-50 \%$ to $+50 \%$. Error

$(\%)$ in the estimated speed for variation of: (a) rotor time constant, (b) stator transient inductance, (c) mutual inductance and (d) stator resistance.

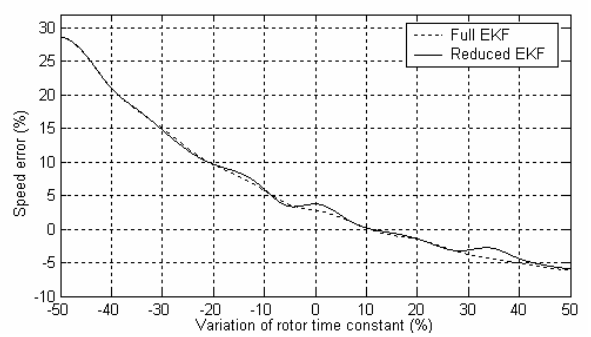

(a)

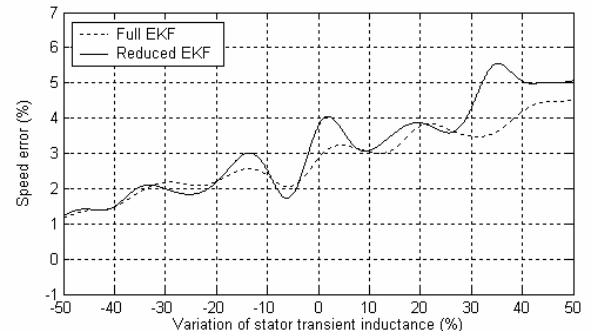

(b)

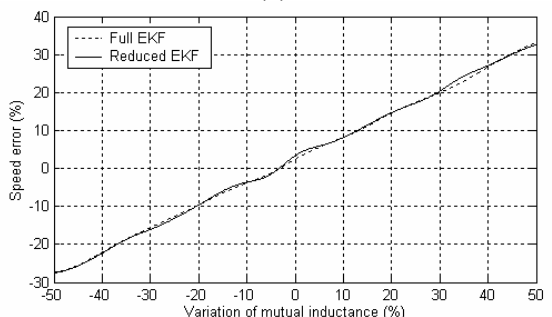

(c)

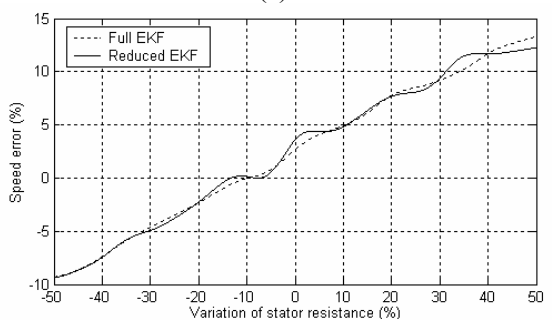

(d)

Fig. 4. The same of fig. 3 but with the motor running at a constant speed of $100 \mathrm{rpm}$.

\section{E. Comparison in general dynamic conditions}

The performance of both EKF algorithms can be compared by the analysis of fig. 5 . A period of 40 seconds is shown in fig. 5(a) with a series of reversals between different speeds values and steady-state zones, including nominal, low as well as zero speed zones.

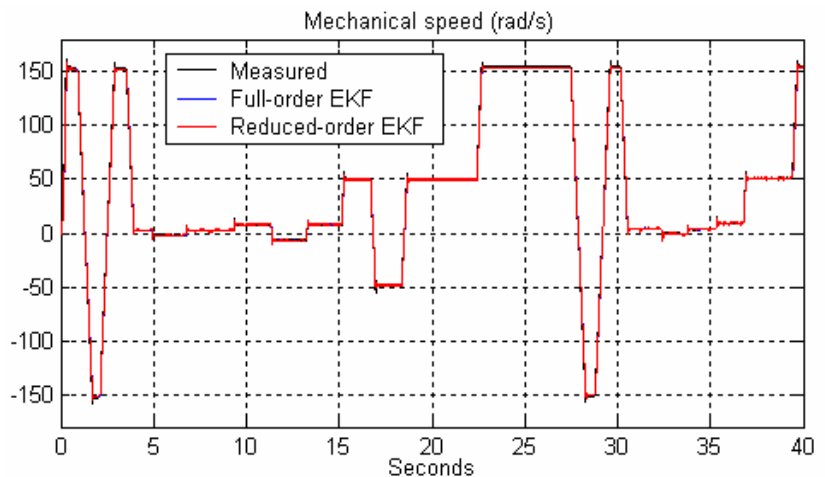

(a) 


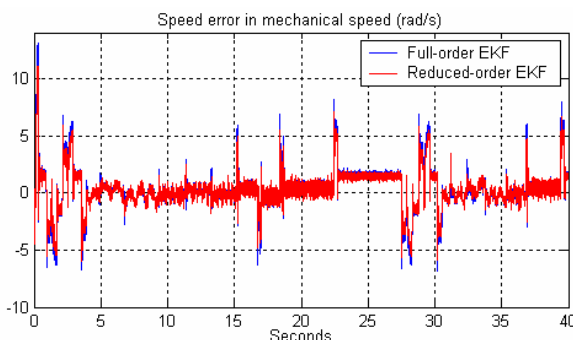

(b)

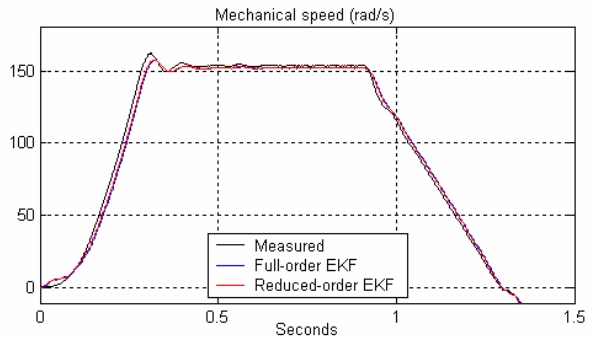

(c)

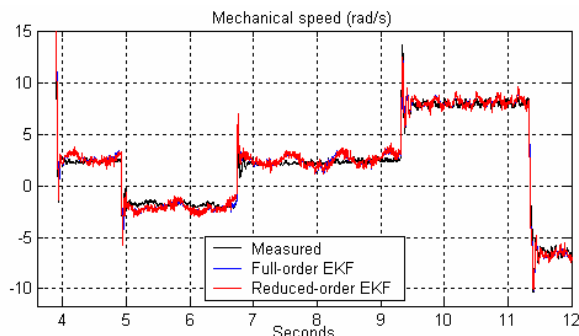

(d)

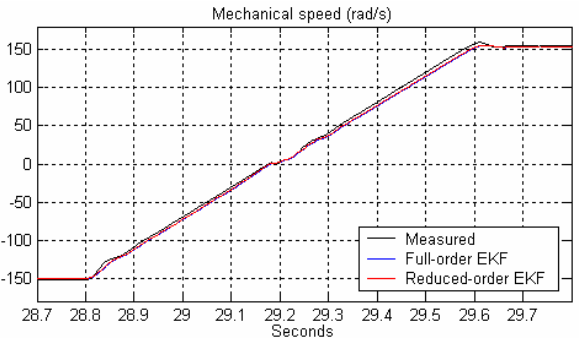

(e)

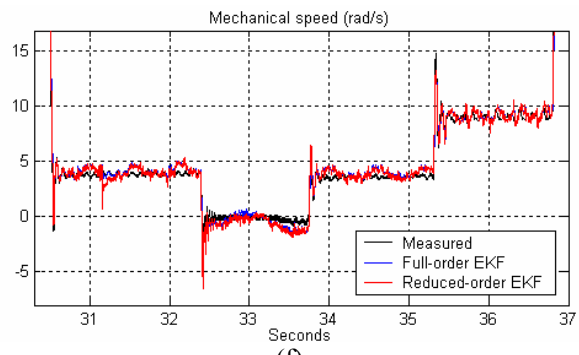

(f)

Fig. 5. Performance of the EKF algorithms in general dynamic conditions: (a) comparison in a series of transients and steady-state zones during 40 seconds; (b) absolute errors in rad/s; (c) to (f) magnifications of (a).

Fig. 5(b) shows the absolute errors of the estimated speeds in $\mathrm{rad} / \mathrm{s}$. Some details are focused from fig. 5(c) to fig. 5(f), with magnifications of fig. 5(a). As can be seen the performance of the reduced order EKF is as good as the full order one either in transient or in steady-state operation. Fig. 6 shows the performance of both EKF algorithms in continuous dynamic operation. The speed reference consists of a square waveform with frequency about $1.7 \mathrm{~Hz}$. In fig. 7 the speed reference (square wave with period of $4 \mathrm{~s}$ and 30 rpm of amplitude), measured and estimated speeds are shown. The load torque is about $10 \mathrm{Nm}$.

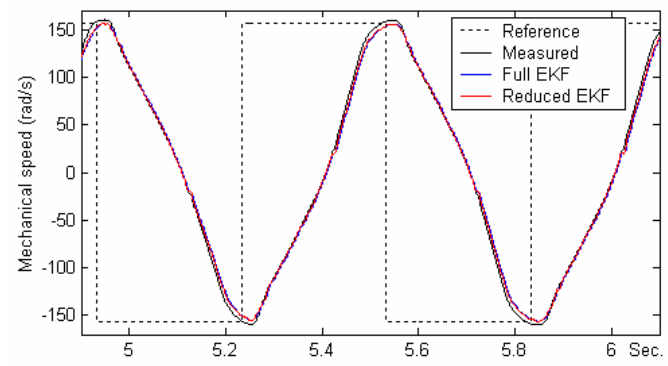

Fig. 6. Performance of the EKF algorithms with speed reversals between $\pm 1500 \mathrm{rpm}$. The frequency of the speed reference is $1.7 \mathrm{~Hz}$.

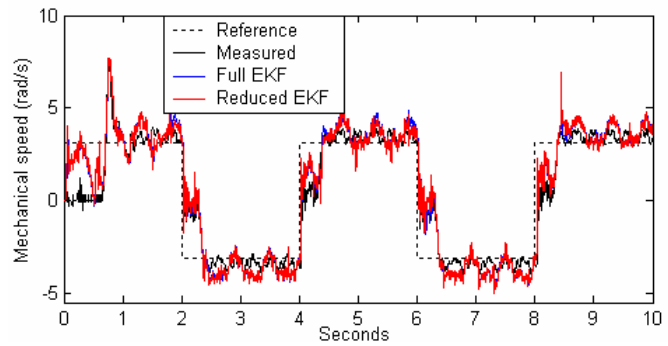

Fig. 7. Performance of the EKF algorithms with speed reversals between $\pm 30 \mathrm{rpm}$. The period of speed reference is $4 \mathrm{~s}$ and the load torque is $10 \mathrm{Nm}$.

\section{F. Comparison of the execution times}

The execution times (in $\mu \mathrm{s}$ ) of both EKF algorithms are presented in some detail in table I. In this case the absolute times are not the most important since they can be reduced if the EKF algorithms are implemented in a low level programming language instead of a high level one such as Simulink. Moreover, they also depend on the hardware. The EKF algorithms were implemented using the DS1103 controller board from dSPACE company. What is important here is to compare the difference between the execution time of the reduced order EKF with the full order one. The steps referred in the first column of table I are shown in the appendix section. It becomes clear from the analysis of table I that the global computation effort is strongly reduced from $14.2 \mu \mathrm{s}$ with the full order EKF to $7.6 \mu \mathrm{s}$ with the reduced order EKF.

TABLE I

Execution times of the EKF algorithms.

\begin{tabular}{rcc}
\hline \hline & \multicolumn{2}{c}{ Execution times $(\mu \mathrm{s})$} \\
\cline { 2 - 3 } Blocks of the EKF & Full order & Reduced order \\
\hline \hline Steps: 1 and 2 & 1.6 & 0.48 \\
\hline Steps: 4 and 6 & 0.9 & 0.12 \\
\hline Steps: 3 and 5 & 4 & 11.2 \\
\hline Steps: 7 and 8 & 1.1 & 2.4 \\
\hline \hline Total: & 7.6 & 14.2 \\
\hline \hline
\end{tabular}

\section{G. Convergence limits relatively to errors in parameters}

The range of parameter variation that keeps the convergence of the algorithms was also compared and the results are shown in table II. All parameters, each one in its 
turn, were varied during real-time operation under the worst conditions, that is to say, with continuous reversals between $\pm 100 \mathrm{rpm}$ with nominal torque. The behaviors of both algorithms are similar. However, the reduced order EKF tends to diverge easier than the full order one above the higher limits of stator transient inductance, mutual inductance and stator resistance. The stator transient inductance is used directly in (3b) whereas in (1a) the inverse value is used. This justifies the difference in table II.

TABLE II

Parameter variation that keeps the convergence of the algorithms with continuous reversals between $\pm 100 \mathrm{rpm}$ with nominal torque.

\begin{tabular}{ccc}
\hline \hline & \multicolumn{2}{c}{ Range of variation } \\
\cline { 2 - 3 } Parameter & Full order EKF & Reduced order EKF \\
\hline \hline$\tau_{\mathrm{r}}(\mathrm{ms})$ & $40 \rightarrow>1000$ & $40 \rightarrow>1000$ \\
\hline$L_{s}{ }^{\prime}(\mathrm{mH})$ & $>0 \rightarrow>80$ & $0 \rightarrow 50$ \\
\hline$L_{M}(\mathrm{mH})$ & $0 \rightarrow 350$ & $0 \rightarrow 350$ \\
\hline$R_{S}(\Omega)$ & $0.2 \rightarrow 3.4$ & $0 \rightarrow 3.4$ \\
\hline \hline
\end{tabular}

\section{CONCLUSION}

A new approach for robust speed estimation in highperformance induction motor drives, namely, in sensorless control, was presented. It is based on a reduced order EKF algorithm and on an innovative methodology used in the state-space model discretization. A comparative study between full and reduced order EKF algorithms was presented. A series of simulation and experimental tests have been achieved using both EKF algorithms. The results have demonstrated a good performance of the reduced order EKF algorithm with respect to the parameter's variation and noise in the full range of load torque and speed. The performance of the new EKF algorithm has proved to be as good as the one obtained with the full order EKF. Some difficulties appear at very low speeds (below a few hundreds of rpm), as it happens with the full order EKF, where the error in the estimated speed can become significant if the parameters are not precisely known, mainly for the mutual inductance. A series of real-time tests using the dSPACE ACE kit based on DS1103 controller board proved the conclusions of the simulation research and some experimental results were presented.

The main conclusion and contribution of this work is that the well-known drawbacks of the EKF, like heavy computational effort for real-time applications, complexity and hard tuning of noise covariance matrices are widely overcome using the proposed reduced order EKF. Very important and practical aspects and new improvements were introduced that strongly reduce the execution time of the new algorithm and simplify the tuning of covariance matrices, since the number of elements to be adjusted is reduced. In fact, the execution time of the new algorithm is about half of the full order EKF and the tuning of the algorithm is not hard any more when compared with the full order EKF and no more complex and time-consuming procedures are required for this purpose as suggested in [6].

\section{APPENDIX}

Steps of the EKF algorithm:

$$
\begin{aligned}
& \text { 1. } \hat{x}(k+1 \mid k)=f(\hat{x}(k \mid k), u(k)) \\
& \text { 2. } F(k)=\left.\frac{\partial f(x(k), u(k))}{\partial x^{T}(k)}\right|_{x(k)=\hat{x}(k \mid k)} \\
& \text { 3. } \hat{P}(k+1 \mid k)=F(k) \hat{P}(k \mid k) F^{T}(k)+Q \\
& \text { 4. } H(k)=\left.\frac{\partial h(x(k))}{\partial x^{T}(k)}\right|_{\hat{x}(k+1 \mid k)} \\
& \text { 5. } K(k+1)=\frac{\hat{P}(k+1 \mid k) H^{T}(k)}{H(k) \hat{P}(k+1 \mid k) H^{T}(k)+R_{m}} \\
& \text { 6. } \varepsilon(k+1)=y(k+1)-h(\hat{x}(k+1 \mid k), k) \\
& \text { 7. } \hat{x}(k+1 \mid k+1)=\hat{x}(k+1 \mid k)+K(k+1) \varepsilon(k+1) \\
& \text { 8. } \hat{P}(k+1 \mid k+1)=[I-K(k+1) H(k)] \hat{P}(k+1 \mid k) \\
& \text { 9. Go to step } 1
\end{aligned}
$$

\section{REFERENCES}

[1] K. Rajashekara, A. Kawamura, K. Maytsuse, Sensorless Control of AC Motor Drives - Speed and Position Sensorless Operation, IEEE Press, 1996.

[2] P. Vas, Sensorless Vector and Direct Torque Control, Oxford University Press, New York, 1998.

[3] B. K. Bose, Modern Power Electronics and AC Drives, Prentice Hall PTR, Upper Saddle River: 2002.

[4] M. Rashed, F. Stronach, P. Vas, "A Stable MRAS-Based Sensorless Control Induction Motor Drive at Low Speeds", in Proc. of the IEEEIEMDC Conference, 2003, in CD-ROM.

[5] D. Drevenšek,D. Žarko, T. Lipo, "A study of Sensorless Control of Induction Motor at Zero Speed Utilizing High Frequency Voltage Injection", EPE Journal, vol. 13, n. ${ }^{\circ} 3$, Aug. 2003, pp. 7-11.

[6] K. L. Shi, T. F. Shan, Y. K. Wong, S. L. Ho, "Speed Estimation of an Induction Motor Drive Using an Optimized Extended Kalman Filter", IEEE Trans. Ind. Electron., vol. 49, n. ${ }^{\circ}$ 1, Feb. 2002, pp. 124-133.

[7] Y. R. Kim, S. K.. Sul, M. H. Park, "Speed Sensorless Vector Control of Induction Motor Using Extended Kalman Filter", in Sensorless Control of AC motor Drives, IEEE Press, New York: 1996, pp. 215223.

[8] E. Levi, M. Wang, "A Speed Estimator for High Performance Sensorless Control of Induction Machines Above Base Speed", in Proc. of the EPE-PEMC Conference, 2002, in CD-ROM.

[9] J. K. Al-Tayie, P.P. Acarnley, "Estimation of Speed, Stator Temperature and Rotor Temperature in Cage Induction Motor Drive Using the Extended Kalman Filter Algorithm", IEE Proc.-Electr. Pawer Appl., vol. 144, n. ${ }^{\circ}$ 5, Sep., 1997, pp. 301-309.

[10] Sensorless Control with Kalman Filter on TMS320 Fixed-Point DSP, Texas Instruments Europe, Literature n. ${ }^{\circ}$ BPRA057, July, 1997.

[11] P. Vas, Parameter Estimation, Condition Monitoring, and Diagnosis of Electrical Machines, Oxford University Press, New York: 1993.

[12] F. L. Lewis, Applied Optimal Control Estimation - Digital Design and Implementation, Prentice Hall, New York: 1992.

[13] A. J. L. Harrison, D. P. Stoten, "Generalized Finite Difference Methods for Optimal Estimation of Derivatives in real-Time Control Problems", in Proc. Instn Mech Engrs, vol. 209, 1995, pp 67-78.

[14] A. V. T. Leite, R. M. E. Araújo, D. R. S. Freitas, "A New Online Identification Methodology for Flux and Parameters Estimation of Vector Controlled Induction Motors", in Proc. of the IEEE-IEMDC 2003 Conference, 2003, in CD-ROM.

[15] A. V. T. Leite, R. M. E. Araújo, D. R. S. Freitas, "A Boot-Strap Estimator for Joint Flux and Parameters Online Identification for Vector Controlled Induction Motor Drives", in Proc. of the EPE 2003 Conference, 2003, in CD-ROM. 